\title{
LEAST-SQUARES FINITE ELEMENT APPROXIMATIONS TO SOLUTIONS OF INTERFACE PROBLEMS*
}

\author{
YANZHAO $\mathrm{CAO}^{\dagger}$ AND MAX D. GUNZBURGER $\ddagger$
}

\begin{abstract}
A least-squares finite element method for second-order elliptic boundary value problems having interfaces due to discontinuous media properties is proposed and analyzed. Both Dirichlet and Neumann boundary data are treated. The boundary value problems are recast into a firstorder formulation to which a suitable least-squares principle is applied. Among the advantages of the method are that nonconforming, with respect to the interface, approximating subspaces may be used. Moreover, the grids used on each side of an interface need not coincide along the interface. Error estimates are derived that improve on other treatments of interface problems and a numerical example is provided to illustrate the method and the analyses.
\end{abstract}

Key words. least-squares finite element methods, interface problems

AMS subject classification. 65 N30

PII. S0036142996303249

1. Introduction. Least-squares finite element methods are the subject of much current interest; a small sample of the recent literature is given by [1], [4], [5], [6], [7], [8], [9], [10], [11], [12], [13], [14]. The obvious advantages of this class of methods is that the discrete problems one must solve are symmetric and positive definite. However, the practicality of these methods is still not fully documented due to a lack of study of the behavior of the methods in the presence of "difficulties" arising from, for example, the use of low-order piecewise polynomial spaces, the application of mixed Dirichlet-Neumann boundary conditions, the discretization of nonconvex polygonal domains, and the need to conserve some global quantity such as mass. Some of these issues were addressed from a computational point of view in [12]. The purpose of this paper is to address another difficulty by defining and analyzing a least-squares finite element method for second-order elliptic equations with discontinuous coefficients; more specifically, we consider interface problems.

One of the first finite element methods (not of least-squares type) treating interface problems was proposed in [2], and a survey of finite element methods for such problems can be found in [3]. In [1], a least-squares method for the interface problem of Poisson equations is introduced after a general theory of the least-squares method has been developed. The authors of [1] were well aware that proving error estimates for the method is difficult, and therefore the weights they use in the terms related to the interface conditions cannot be rigorously justified. In this paper, following the approach of [8] and [13], we formulate the problem as a first-order system and then apply least-squares principles to this system. The theory of [8] can also be applied to the interface problem. However, the error estimate there requires that solutions be sufficiently smooth, which may not be true for interface problems. Least-squares finite element methods for interface problems are also considered in [15].

\footnotetext{
*Received by the editors May 8, 1996; accepted for publication (in revised form) October 31, 1996.

http://www.siam.org/journals/sinum/35-1/30324.html

${ }^{\dagger}$ Department of Mathematics, Virginia Tech, Blacksburg, VA 24061-0123 (yanzhao@math.vt.edu). This research was supported by Air Force Office of Scientific Research grant AFOSR-93-1-0280.

‡Department of Mathematics, Iowa State University, Ames IA 50011-2064 (gunzburg@ iastate.edu.edu/gunzburg). This research was supported by Air Force Office of Scientific Research grant AFOSR-93-1-0280.
} 
To avoid global regularity requirements, we introduce two terms in the leastsquares functional that are related to the conditions on the interface. Our error analysis shows that the method has nearly optimal order of accuracy with respect to an appropriately defined norm. The weights used for these terms are justified by the error estimate and are supported by our numerical experiments.

The paper is organized as follows. In the next section we introduce the problem and some necessary notations. An existence and uniqueness theorem is stated. Then, in section 3 we define and analyze the least-squares finite element method for the case of Dirichlet boundary conditions. A coercive property for the least-squares functional is proved and error estimates are obtained. In section 4 we extend the analyses to problems with inhomogeneous Neumann boundary conditions. Finally, in section 5, a computational example is presented.

In order to keep the exposition simple, our discussion is in the context of a single interface separating two subdomains in each of which the coefficients of the partial differential equations are "smooth." However, our algorithms and results extend in an obvious manner to problems with multiple interfaces and domains, so long as the assumed regularity results within the subdomains separated by the interfaces remain valid. In particular, we will assume that each of the subdomains has a "smooth" boundary or, in the very special situations for which this can be arranged, each has a convex boundary.

2. Statement of the problem. Assume that $\Omega$ is an open bounded domain in $\mathbb{R}^{n}, n=2$ or 3 , with smooth boundary. $\Omega_{1}$ and $\Omega_{2}$ are two open subsets of $\Omega$ such that $\bar{\Omega}=\bar{\Omega}_{1} \cup \bar{\Omega}_{2}, \Omega_{1} \cap \Omega_{2}=\emptyset$. Let $\Gamma_{0}=\partial \Omega, \Gamma=\bar{\Omega}_{1} \cap \bar{\Omega}_{2}, \Gamma_{1}=\partial \Omega \cap \bar{\Omega}_{1}$, and $\Gamma_{2}=\partial \Omega \cap \bar{\Omega}_{2}$. Here, $\Gamma$ is referred to as the interface. Throughout, we assume that the subdomains $\Omega_{1}$ and $\Omega_{2}$ both have smooth (or in very special situtations, convex) boundaries. Smooth boundaries can occur, for example, if $\bar{\Omega}_{1} \subset \Omega, \Omega_{2}=\Omega-\bar{\Omega}_{1}$, and $\Omega_{1}$ has a smooth boundary. Convex subdomains result, for example, if one subdivides a rectangle into smaller rectangles.

Consider the following elliptic boundary value problem on $\Omega$ :

$$
\begin{gathered}
-\operatorname{div}\left(A_{i} \nabla u_{i}\right)+c_{i} u_{i}=f_{i} \quad \text { in } \Omega_{i}, i=1,2, \\
\left.u_{i}\right|_{\Gamma_{i}}=0, \quad i=1,2, \\
\left.u_{1}\right|_{\Gamma}=\left.u_{2}\right|_{\Gamma}, \quad \text { and }\left.\quad\left(A_{1} \nabla u_{1} \cdot \mathbf{n}_{1}\right)\right|_{\Gamma}+\left.\left(A_{2} \nabla u_{2} \cdot \mathbf{n}_{2}\right)\right|_{\Gamma}=0,
\end{gathered}
$$

where $c_{i} \geq c>0$ and $A_{i}=\left(a_{l k}^{i}\right), i=1,2$, and $l, k=1, \ldots, n$, are $n \times n$ positive definite matrices so that, if $\lambda_{j}^{i}, j=1, \ldots, n$, denote the eigenvalues of $A_{i}$, then there exist two constants $C_{a}$ and $C_{b}$ such that

$$
0<C_{a} \leq \lambda_{j}^{i} \leq C_{b}, \quad i=1,2, j=1, \ldots, n .
$$

The cases for which $c_{1}=0$ and/or $c_{2}=0$ may also be treated at the expense of greatly complicating the analyses. The constants appearing in our estimates will, in general, depend on $C_{a}$ and $C_{b}$ and, in particular, on the ratio $C_{b} / C_{a}$. In $(2.3), \mathbf{n}_{i}$ denotes the unit outer normal vector on $\Omega_{i}, i=1,2$.

For $k \geq 0$, we denote by $H^{k}(\mathcal{D})$ the standard Sobolev space consisting of functions defined over the domain $\mathcal{D}$ and having square integrable derivatives of order up to $k$. For negative values of $k$, these spaces are also defined in the usual manner as appropriate dual spaces. In particular, $H^{-1}(\mathcal{D})$ is the dual space of $H_{0}^{1}(\mathcal{D})$, where the 
latter is the space of functions having one square integrable derivative with respect to $\mathcal{D}$ and that vanish on the boundary of that domain. Also, $\mathbf{H}^{k}(\mathcal{D})=\left(H^{k}(\mathcal{D})\right)^{n}$ denotes the space of vector-valued functions, each of whose components belongs to $H^{k}(\mathcal{D})$. The standard Sobolev norm for functions belonging to $H^{k}(\mathcal{D})$ and $\mathbf{H}^{k}(\mathcal{D})=\left(H^{k}(\mathcal{D})\right)^{n}$ is denoted by $\|\cdot\|_{k, \mathcal{D}}$.

For $k \geq 0$, define the Banach spaces

$$
\dot{H}^{k}(\Omega)=\left\{u=\left(u_{1}, u_{2}\right) \quad\left|\quad u_{i}=u\right|_{\Omega_{i}} \in H^{k}\left(\Omega_{i}\right), \quad i=1,2\right\}
$$

with norm $\|u\|\left\|_{k}=\right\| u_{1}\left\|_{k, \Omega_{1}}+\right\| u_{2} \|_{k, \Omega_{2}}$ and

$$
\dot{\mathbf{V}}^{k}(\Omega)=\left\{\mathbf{v}=\left(\mathbf{v}_{1}, \mathbf{v}_{2}\right) \quad\left|\quad \mathbf{v}_{i}=\mathbf{v}\right|_{\Omega_{i}} \in \mathbf{H}^{k}\left(\Omega_{i}\right), \quad i=1,2\right\}
$$

with norm $\|\mathbf{v}\|\left\|_{k}=\right\| \mathbf{v}_{1}\left\|_{k, \Omega_{1}}+\right\| \mathbf{v}_{2} \|_{k, \Omega_{2}}$. We may extend these definitions to $k=-1$; for example,

$$
\dot{H}^{-1}(\Omega)=\left\{u=\left(u_{1}, u_{2}\right) \quad\left|\quad u_{i}=u\right|_{\Omega_{i}} \in H_{\Gamma_{i}}^{-1}\left(\Omega_{i}\right), \quad i=1,2\right\},
$$

where $H_{\Gamma_{i}}^{-1}\left(\Omega_{i}\right)$ denotes the dual space of $H_{\Gamma_{i}}^{1}\left(\Omega_{i}\right)=\left\{u \in H^{1}\left(\Omega_{i}\right) \mid u=0\right.$ on $\left.\Gamma_{i}\right\}$, $i=1,2$. Note that, generally, $\dot{H}^{k}(\Omega) \not \subset H^{k}(\Omega)$ and $\dot{\mathbf{V}}^{k}(\Omega) \not \subset \mathbf{V}^{k}(\Omega)$. In particular, we will work with the space $\dot{H}^{1}(\Omega)$, which is generally not a subspace of $H^{1}(\Omega)$, so that approximations for $\left\{u|u|_{\Omega_{i}}=u_{i}, i=1,2\right\}$ will be nonconforming in the sense that these approximations need not belong to $H^{1}(\Omega)$.

Let

$$
H=\dot{H}^{1}(\Omega), \quad \mathbf{V}=\dot{\mathbf{V}}^{1}(\Omega), \quad \text { and } \quad H_{0}=\left\{u \in H|u|_{\Gamma_{0}}=0\right\} .
$$

Along the interface $\Gamma$, let $[u]_{\Gamma}=u_{1}-u_{2}$ and $[\mathbf{v} \cdot \mathbf{n}]_{\Gamma}=\mathbf{v}_{1} \cdot \mathbf{n}_{1}+\mathbf{v}_{2} \cdot \mathbf{n}_{2}$. Let

$$
H(\Gamma, \Omega)=\left\{u \in H \quad \mid \quad[u]_{\Gamma}=0\right\}, \quad H_{0}(\Gamma, \Omega)=H(\Gamma, \Omega) \cap H_{0},
$$

and

$$
\mathbf{V}(\Gamma, \Omega)=\left\{\mathbf{v} \in \mathbf{V} \quad \mid \quad[\mathbf{v} \cdot \mathbf{n}]_{\Gamma}=0\right\} .
$$

Note that $H(\Gamma, \Omega) \subset H^{1}(\Omega)$. Also, define the Hilbert spaces

$$
\mathbf{V}\left(\operatorname{div}, \Omega_{i}\right)=\left\{\mathbf{v} \in \mathbf{L}_{2}\left(\Omega_{i}\right)=\left(L_{2}\left(\Omega_{i}\right)\right)^{n} \quad \mid \quad \operatorname{div} \mathbf{v} \in L_{2}\left(\Omega_{i}\right)\right\}
$$

with norm $\|\mathbf{v}\|_{\operatorname{div}, \Omega_{i}}=\|\mathbf{v}\|_{0, \Omega_{i}}+\|\operatorname{div} \mathbf{v}\|_{0, \Omega_{i}}$ and

$$
\mathbf{V}(\operatorname{div})=\left\{\mathbf{v}=\left(\mathbf{v}_{\mathbf{1}}, \mathbf{v}_{\mathbf{2}}\right) \quad \mid \quad \mathbf{v}_{i} \in \mathbf{V}\left(\operatorname{div}, \Omega_{i}\right)\right\}
$$

with norm $\|\mathbf{v}\|\left\|_{\text {div }}=\right\| \mathbf{v}_{1}\left\|_{\operatorname{div}, \Omega_{1}}+\right\| \mathbf{v}_{2} \|_{\text {div }, \Omega_{2}}$.

Concerning the problem (2.1)-(2.3), we have the following result.

ThEOREM 2.1. Assume, for $k \geq 1$, that $f \in \dot{H}^{k-2}(\Omega), a_{l j}^{i} \in H^{k}\left(\bar{\Omega}_{i}\right), i=1,2$, $l, j=1, \ldots, n$, and $c_{i} \in H^{k}\left(\bar{\Omega}_{i}\right)$ for $i=1,2$. Then, there exists a unique solution $u \in \dot{H}^{k}(\Omega)$ for $(2.1)-(2.3)$.

Proof. See [16]. 
3. Least-squares finite element approximations. We rewrite (2.1)-(2.3) as a system of first-order differential equations:

$$
\begin{gathered}
-\operatorname{div}\left(\mathbf{v}_{i}\right)+c_{i} u_{i}=f \quad \text { in } \Omega_{i}, \quad i=1,2, \\
A_{i} \nabla u_{i}-\mathbf{v}_{i}=0 \quad \text { in } \Omega_{i}, \quad i=1,2, \\
\left.u\right|_{\Gamma_{0}}=0, \quad[u]_{\Gamma}=0, \quad \text { and } \quad[\mathbf{v} \cdot \mathbf{n}]_{\Gamma}=0 .
\end{gathered}
$$

We introduce subspaces $H^{h} \subset H_{0}$ and $\mathbf{V}^{h} \subset \mathbf{V}$ parameterized by $h$, usually chosen to be some measure of the grid size such as the largest diameter of the triangles in a triangulation of $\Omega$. Note that $H^{h}$ need not be a subset of $H^{1}(\Omega)$ so that in this sense our method is nonconforming.

We assume that the subspaces $H^{h}$ and $\mathbf{V}^{h}$ possess the approximation properties

$$
\inf _{u_{h} \in H^{h}}\left\|u-u_{h}\right\|_{k, \Omega_{i}} \leq C h^{s-k}\|u\|_{s, \Omega_{i}} \quad \forall u \in \dot{H}^{s}(\Omega), u^{h} \in H^{h}, i=1,2
$$

and

$$
\inf _{\mathbf{v}^{h} \in \mathbf{V}^{h}}\left\|\mathbf{v}-\mathbf{v}^{h}\right\|_{k, \Omega_{i}} \leq h^{s-k}\|\mathbf{v}\|_{s, \Omega_{i}} \quad \forall \mathbf{v} \in \dot{\mathbf{V}}^{s}(\Omega), \mathbf{v}^{h} \in \mathbf{V}^{h}, i=1,2,
$$

where $0<k<s$. As a result, we have that

$$
\inf _{u_{h} \in H^{h}}\left\|u-u_{h}\right\|_{k} \leq C h^{s-k}\|u\|_{s} \quad \forall u \in \dot{H}^{s}(\Omega), u^{h} \in H^{h}
$$

and

$$
\inf _{\mathbf{v}^{h} \in \mathbf{V}^{h}} \mid\left\|\mathbf{v}-\mathbf{v}^{h}\right\|_{k} \leq h^{s-k}\|\mathbf{v}\|_{s} \quad \forall \mathbf{v} \in \dot{\mathbf{V}}^{s}(\Omega), \mathbf{v}^{h} \in \mathbf{V}^{h},
$$

where $0<k<s$. We also assume that the following inverse inequality holds in $H^{h}$ : there exists a constant $C$ such that for $u^{h}=\left(u_{1}^{h}, u_{2}^{h}\right) \in H^{h}$,

$$
\left\|u_{1}^{h}-u_{2}^{h}\right\|_{1 / 2, \Gamma} \leq \frac{C}{h^{1 / 2}}\left\|u_{1}^{h}-u_{2}^{h}\right\|_{0, \Gamma} .
$$

Note that if the restrictions to $\Gamma$ of the approximating spaces in $\Omega_{1}$ and $\Omega_{2}$ coincide, then the inverse property (3.6) is simply the inverse property in the usual sense.

3.1. The least-squares functional. We define a functional on $H_{0} \times \mathbf{V}$ as follows. For $u \in H_{0}$ and $\mathbf{v} \in \mathbf{V}$, let

$$
\begin{array}{r}
\mathcal{J}(u, \mathbf{v} ; f)=\sum_{i=1}^{2}\left(\left\|-\operatorname{div}_{i}+c_{i} u_{i}-f\right\|_{0}^{2}+\left\|A_{i} \nabla u_{i}-\mathbf{v}_{i}\right\|_{0}^{2}\right) \\
+\frac{1}{h^{1+\epsilon_{0}}} \int_{\Gamma}[u]_{\Gamma}^{2} d \Gamma+\frac{1}{h^{\epsilon_{1}}} \int_{\Gamma}[\mathbf{v} \cdot \mathbf{n}]_{\Gamma}^{2} d \Gamma,
\end{array}
$$

where $f \in \dot{H}^{0}(\Omega)$ and $\epsilon_{0}, \epsilon_{1}>0$. Note that if $u \in H_{0}(\Gamma, \Omega)$ and $\mathbf{v} \in \mathbf{V}(\Gamma, \Omega)$, then the last two terms in (3.7) vanish. Also, note that if $u$ and $\mathbf{v}$ satisfy (3.1)-(3.3), then $\mathcal{J}(u, \mathbf{v} ; f)=0$.

The functional $\mathcal{J}(\cdot, \cdot ; \cdot)$ satisfies the following coercivity property. 
Proposition 3.1. Let $u_{h}=\left(u_{1}^{h}, u_{2}^{h}\right) \in H^{h}, \mathbf{v}^{h}=\left(\mathbf{v}_{1}^{h}, \mathbf{v}_{2}^{h}\right) \in \mathbf{V}^{h}, u=\left(u_{1}, u_{2}\right) \in$ $H_{0}(\Gamma, \Omega)$, and $\mathbf{v}=\left(\mathbf{v}_{1}, \mathbf{v}_{2}\right) \in \mathbf{V}(\Gamma, \Omega)$. Then, for $h$ sufficiently small, there exists a constant $C>0$ independent of $h$ such that

$$
\mathcal{J}\left(u-u^{h}, \mathbf{v}-\mathbf{v}^{h} ; 0\right) \geq C\left(\left\|u-u^{h}\right\|_{1}^{2}+\left\|\mathbf{v}-\mathbf{v}^{h}\right\|_{\text {div }}^{2}\right) .
$$

Proof. Let

$$
\begin{gathered}
\widehat{\mathcal{J}}(u, \mathbf{v})=\sum_{i=1}^{2}\left(\left\|-c_{i}^{-1 / 2} \operatorname{div} \mathbf{v}_{i}+c_{i}^{1 / 2} u_{i}\right\|_{0}^{2}+\left\|A_{i}^{\frac{1}{2}} \nabla u_{i}-A_{i}^{-\frac{1}{2}} \mathbf{v}_{i}\right\|_{0}^{2}\right) \\
+\frac{1}{h^{1+\epsilon_{0}}} \int_{\Gamma}[u]_{\Gamma}^{2} d \Gamma+\frac{1}{h^{\epsilon_{1}}} \int_{\Gamma}[\mathbf{v} \cdot \mathbf{n}]_{\Gamma}^{2} d \Gamma .
\end{gathered}
$$

It is easy to see that $\mathcal{J}(\cdot, \cdot ; 0)$ and $\widehat{\mathcal{J}}(\cdot, \cdot)$ are equivalent, i.e., that there exist two positive constants $C_{1}$ and $C_{2}$ such that

$$
C_{1} \mathcal{J}(u, \mathbf{v} ; 0) \leq \widehat{\mathcal{J}}(u, \mathbf{v}) \leq C_{2} \mathcal{J}(u, \mathbf{v} ; 0)
$$

for all $u \in H$ and $\mathbf{v} \in \mathbf{V}$. Thus, it suffices to prove that

$$
\widehat{\mathcal{J}}\left(u-u^{h}, \mathbf{v}-\mathbf{v}^{h}\right) \geq C\left(\left\|u-u^{h}\right\|_{1}^{2}+\left\|\mathbf{v}-\mathbf{v}^{h}\right\|_{\text {div }}^{2}\right)
$$

for some constant $C>0$. Now, since $u \in H_{0}(\Gamma, \Omega)$ and $\mathbf{v} \in \mathbf{V}(\Gamma, \Omega)$, we have that

$$
\begin{aligned}
\widehat{\mathcal{J}}\left(u-u^{h}, \mathbf{v}-\mathbf{v}^{h}\right) & \\
=\sum_{i=1}^{2}(\| & -c_{i}^{-1 / 2} \operatorname{div}\left(\mathbf{v}_{i}-\mathbf{v}_{i}^{h}\right)+c_{i}^{1 / 2}\left(u_{i}-u_{i}^{h}\right) \|_{0}^{2} \\
& \left.+\left\|A_{i}^{1 / 2} \nabla\left(u_{i}-u_{i}^{h}\right)-A_{i}^{-1 / 2}\left(\mathbf{v}_{i}-\mathbf{v}_{i}^{h}\right)\right\|_{0}^{2}\right) \\
& +\frac{1}{h^{1+\epsilon_{0}}} \int_{\Gamma}\left|u_{1}^{h}-u_{2}^{h}\right|^{2} d \Gamma+\frac{1}{h^{\epsilon_{1}}} \int_{\Gamma}\left(\left(\mathbf{v}_{1}^{h}-\mathbf{v}_{2}^{h}\right) \cdot \mathbf{n}\right)^{2} d \Gamma .
\end{aligned}
$$

Integrating by parts, one obtains, for $i=1,2$,

$$
\begin{gathered}
\left\|-c_{i}^{-1 / 2} \operatorname{div}\left(\mathbf{v}_{i}-\mathbf{v}_{i}^{h}\right)+c_{i}^{1 / 2}\left(u_{i}-u_{i}^{h}\right)\right\|_{0}^{2}+\left\|A_{i}^{1 / 2} \nabla\left(u_{i}-u_{i}^{h}\right)-A_{i}^{-1 / 2}\left(\mathbf{v}_{i}-\mathbf{v}_{i}^{h}\right)\right\|_{0}^{2} \\
=\left\|c_{i}^{-1 / 2} \operatorname{div}\left(\mathbf{v}_{i}-\mathbf{v}_{i}^{h}\right)\right\|_{0}^{2}+\left\|c_{i}^{1 / 2}\left(u_{i}-u_{i}^{h}\right)\right\|_{0}^{2}-2 \int_{\Omega_{i}} \operatorname{div}\left(\mathbf{v}_{i}-\mathbf{v}_{i}^{h}\right)\left(u_{i}-u_{i}^{h}\right) d \Omega \\
\quad+\left\|A_{i}^{1 / 2} \nabla\left(u_{i}-u_{i}^{h}\right)\right\|_{0}^{2}+\left\|A_{i}^{-1 / 2}\left(\mathbf{v}_{i}-\mathbf{v}_{i}^{h}\right)\right\|_{0}^{2}-2 \int_{\Omega_{i}} \nabla\left(u_{i}-u_{i}^{h}\right) \cdot\left(\mathbf{v}_{i}-\mathbf{v}_{i}^{h}\right) d \Omega \\
=\left\|c_{i}^{-1 / 2} \operatorname{div}\left(\mathbf{v}_{i}-\mathbf{v}_{i}^{h}\right)\right\|_{0}^{2}+\left\|c_{i}^{1 / 2}\left(u_{i}-u_{i}^{h}\right)\right\|_{0}^{2}+\left\|A_{i}^{1 / 2} \nabla\left(u_{i}-u_{i}^{h}\right)\right\|_{0}^{2} \\
\quad+\left\|A_{i}^{-1 / 2}\left(\mathbf{v}_{i}-\mathbf{v}_{i}^{h}\right)\right\|_{0}^{2}-2 \int_{\Gamma}\left(u_{i}-u_{i}^{h}\right)\left(\mathbf{v}_{i}-\mathbf{v}_{i}^{h}\right) \cdot \mathbf{n} d \Gamma .
\end{gathered}
$$

Hence, for some constant $C_{3}>0$,

$$
\begin{aligned}
\widehat{\mathcal{J}}\left(u-u^{h}, \mathbf{v}-\mathbf{v}^{h}\right) & \\
\geq & C_{3} \sum_{i=1}^{2}\left(\left\|\operatorname{div}\left(\mathbf{v}_{i}-\mathbf{v}_{i}^{h}\right)\right\|_{0}^{2}+\left\|u_{i}-u_{i}^{h}\right\|_{0}^{2}+\left\|\nabla\left(u_{i}-u_{i}^{h}\right)\right\|_{0}^{2}+\left\|\mathbf{v}_{i}-\mathbf{v}_{i}^{h}\right\|_{0}^{2}\right) \\
& +2 \int_{\Gamma}\left(u_{1}-u_{1}^{h}\right)\left(\mathbf{v}_{1}-\mathbf{v}_{1}^{h}\right) \cdot \mathbf{n} d \Gamma-2 \int_{\Gamma}\left(u_{2}-u_{2}^{h}\right)\left(\mathbf{v}_{2}-\mathbf{v}_{2}^{h}\right) \cdot \mathbf{n} d \Gamma \\
& +\frac{1}{h^{1+\epsilon_{0}}} \int_{\Gamma}\left(u_{1}^{h}-u_{2}^{h}\right)^{2} d \Gamma+\frac{1}{h^{\epsilon_{1}}} \int_{\Gamma}\left(\left(\mathbf{v}_{1}^{h}-\mathbf{v}_{2}^{h}\right) \cdot \mathbf{n}\right)^{2} d \Gamma .
\end{aligned}
$$


By the definition of $H_{0}(\Gamma, \Omega)$ and $\mathbf{V}(\Gamma, \Omega)$, trace theorems, and the inverse property (3.6) on $H^{h}$, we have, for some constant $C_{4}>0$,

$$
\begin{aligned}
& \left|\int_{\Gamma}\left(u_{1}-u_{1}^{h}\right)\left(\mathbf{v}_{1}-\mathbf{v}_{1}^{h}\right) \cdot \mathbf{n} d \Gamma-\int_{\Gamma}\left(u_{2}-u_{2}^{h}\right)\left(\mathbf{v}_{2}-\mathbf{v}_{2}^{h}\right) \cdot \mathbf{n} d \Gamma\right| \\
& \quad=\left|\int_{\Gamma}\left(u_{1}^{h}-u_{2}^{h}\right)\left(\mathbf{v}_{1}-\mathbf{v}_{1}^{h}\right) \cdot \mathbf{n} d \Gamma+\int_{\Gamma}\left(u_{2}-u_{2}^{h}\right)\left(\mathbf{v}_{1}^{h}-\mathbf{v}_{2}^{h}\right) \cdot \mathbf{n} d \Gamma\right| \\
& \quad \leq\left\|u_{1}^{h}-u_{2}^{h}\right\|_{1 / 2, \Gamma}\left\|\left(\mathbf{v}_{1}-\mathbf{v}_{1}^{h}\right) \cdot \mathbf{n}\right\|_{-1 / 2, \Gamma}+\left\|u_{2}-u_{2}^{h}\right\|_{0, \Gamma}\left\|\left(\mathbf{v}_{1}^{h}-\mathbf{v}_{2}^{h}\right) \cdot \mathbf{n}\right\|_{0, \Gamma} \\
& \quad \leq C_{4}\left(\epsilon\left\|\mathbf{v}-\mathbf{v}^{h}\right\|_{\operatorname{div}}^{2}+\frac{1}{h \epsilon}\left\|u_{1}^{h}-u_{2}^{h}\right\|_{0, \Gamma}^{2}+\frac{1}{\epsilon}\left\|\mathbf{v}_{1}^{h}-\mathbf{v}_{2}^{h}\right\|_{0, \Gamma}^{2}+\epsilon\left\|u-u^{h}\right\|_{1}^{2}\right) .
\end{aligned}
$$

Hence,

$$
\begin{aligned}
\widehat{\mathcal{J}}\left(u-u^{h}, \mathbf{v}-\mathbf{v}^{h}\right) & \geq\left(C_{3}-C_{4} \epsilon\right)\left(\left\|u-u^{h}\right\|_{1}^{2}+\left\|\mathbf{v}-\mathbf{v}^{h}\right\|_{\text {div }}^{2}\right) \\
& +\left(\frac{1}{h^{1+\epsilon_{0}}}-\frac{2 C_{4}}{h \epsilon}\right) \int_{\Gamma}\left|u_{1}^{h}-u_{2}^{h}\right|^{2} d \Gamma+\left(\frac{1}{h^{\epsilon_{1}}}-\frac{2 C_{4}}{\epsilon}\right) \int_{\Gamma}\left(\left(\mathbf{v}_{1}^{h}-\mathbf{v}_{2}^{h}\right) \cdot \mathbf{n}\right)^{2} d \Gamma .
\end{aligned}
$$

We first choose $\epsilon$ small enough so that $C_{3}-C_{4} \epsilon>0$. Then, for this fixed $\epsilon$, we choose $h_{0}$ sufficiently small so that $2 C_{4} h_{0}^{\epsilon_{0}} \leq \epsilon$ and $2 C_{4} h_{0}^{\epsilon_{1}} \leq \epsilon$. Thus, for $0<h<h_{0}$, we have that

$$
\widehat{\mathcal{J}}\left(u-u^{h}, \mathbf{v}-\mathbf{v}^{h}\right) \geq C\left(\|\| u-u^{h}\left\|_{1}^{2}+\right\| \mathbf{v}-\mathbf{v}^{h} \|_{\text {div }}^{2}\right) .
$$

Proposition 3.1 shows a certain coercive property about the functional $\mathcal{J}$. If we choose $u=0$ and $\mathbf{v}=\mathbf{0}$ in (3.8), then we see that the coercive property is true on the finite-dimensional subspace $H^{h} \times \mathbf{V}^{h}$ of $H \times \mathbf{V}$, i.e., $\mathcal{J}\left(u^{h}, \mathbf{v}^{h} ; 0\right) \geq$ $C\left(\left\|u^{h}\right\|_{1}^{2}+\left\|\mathbf{v}^{h}\right\|_{H(\text { div })}^{2}\right)$ for $\left(u^{h}, \mathbf{v}^{h}\right) \in H^{h} \times \mathbf{V}^{h}$. However, this does not hold for all elements of $H \times \mathbf{V}$. Neverthless, Proposition 3.1 suffices for us to obtain an error estimate for the least-squares finite element approximations of the solution of (3.1)(3.3).

3.2. Finite element approximations. We define $\left(u_{*}^{h}, \mathbf{v}_{*}^{h}\right)$ to be the solution of the following problem:

$$
J\left(u_{*}^{h}, \mathbf{v}_{*}^{h} ; f\right)=\min _{u_{h} \in H^{h}, \mathbf{v}^{h} \in \mathbf{V}^{h}} J\left(u^{h}, \mathbf{v}^{h} ; f\right) .
$$

We then have the following error estimate.

THEOREM 3.2. Let $s>0$. Assume that the solution $(u, \mathbf{v})$ of (3.1)-(3.3) satisfies $u \in \dot{H}^{s+1}(\Omega) \cap H_{0}(\Gamma, \Omega)$ and $\mathbf{v} \in \dot{V}^{s+1}(\Omega) \cap \mathbf{V}(\Gamma, \Omega)$. Then, for $h$ sufficiently small and for $0<\epsilon_{1} \leq 1$ and any $\delta>\epsilon_{0}>0$, there exists a constant $C>0$ such that

$$
\left\|u-u_{*}^{h}\right\|_{1}+\left\|\mathbf{v}-\mathbf{v}_{*}^{h}\right\|_{\text {div }} \leq C h^{s-\delta}\left(\|u\|_{s+1}+\|\mid \mathbf{v}\|_{s+1}\right) .
$$

Proof. By the approximation properties (3.4) and (3.5), there exist $\widehat{u}^{h} \in H^{h}$ and $\widehat{\mathbf{v}}^{h} \in \mathbf{V}^{h}$ such that

$$
\left\|u-\widehat{u}^{h}\right\|_{1} \leq C h^{s}\|u\|_{s+1}
$$

and

$$
\left\|\mathbf{v}-\widehat{\mathbf{v}}^{h}\right\|_{1} \leq C h^{s}\|\mathbf{v}\|_{s+1} .
$$


By Proposition 3.1 and the definition of $u_{*}^{h}$ and $\mathbf{v}_{*}^{h}$,

$$
\begin{aligned}
& \left\|u-u_{*}^{h}\right\|_{1}^{2}+\left\|\mathbf{v}-\mathbf{v}_{*}^{h}\right\|_{\operatorname{div}}^{2} \leq C \mathcal{J}\left(u-u_{*}^{h}, \mathbf{v}-\mathbf{v}_{*}^{h} ; 0\right) \\
& =C \mathcal{J}\left(u_{*}^{h}, \mathbf{v}_{*}^{h} ; f\right) \leq C \mathcal{J}\left(\widehat{u}^{h}, \widehat{\mathbf{v}}^{h} ; f\right)=C \mathcal{J}\left(u-\widehat{u}^{h}, \mathbf{v}-\widehat{\mathbf{v}}^{h} ; 0\right) \\
& \leq\left\|u-\widehat{u}^{h}\right\|_{1}^{2}+\left\|\mathbf{v}-\widehat{\mathbf{v}}^{h}\right\|_{\text {div }}^{2} \\
& \quad+\frac{1}{h^{1+\epsilon_{0}}} \int_{\Gamma}\left(\widehat{u}_{1}^{h}-\widehat{u}_{2}^{h}\right)^{2} d \Gamma+\frac{1}{h^{\epsilon_{1}}} \int_{\Gamma}\left(\left(\widehat{\mathbf{v}}_{1}^{h}-\widehat{\mathbf{v}}_{2}^{h}\right) \mathbf{n}\right)^{2} d \Gamma .
\end{aligned}
$$

Using trace theorems, we have that

$$
\begin{aligned}
\frac{1}{h^{1+\epsilon_{0}}} \int_{\Gamma}\left(\widehat{u}_{1}^{h}-\widehat{u}_{2}^{h}\right)^{2} d \Gamma+\frac{1}{h^{\epsilon_{1}}} \int_{\Gamma}\left(\left(\widehat{\mathbf{v}}_{1}^{h}-\widehat{\mathbf{v}}_{2}^{h}\right) \cdot \mathbf{n}\right)^{2} d \Gamma \\
=\frac{1}{h^{1+\epsilon_{0}}} \int_{\Gamma}\left(\widehat{u}_{1}^{h}-u_{1}+u_{2}-\widehat{u}_{2}^{h}\right)^{2} d \Gamma \\
\quad \quad+\frac{1}{h^{\epsilon_{1}}} \int_{\Gamma}\left(\left(\widehat{\mathbf{v}}_{1}^{h}-\mathbf{v}_{1}+\mathbf{v}_{2}-\widehat{\mathbf{v}}_{2}^{h}\right) \cdot \mathbf{n}\right)^{2} d \Gamma \\
\leq \frac{2}{h^{1+\epsilon_{0}}}\left(\int_{\Gamma}\left(\widehat{u}_{1}^{h}-u\right)^{2} d \Gamma+\int_{\Gamma}\left(\widehat{u}_{2}^{h}-u\right)^{2} d \Gamma\right) \\
\quad+\frac{2}{h^{\epsilon_{1}}}\left(\int_{\Gamma}\left(\left(\widehat{\mathbf{v}}_{1}^{h}-\mathbf{v}\right) \cdot \mathbf{n}\right)^{2} d \Gamma+\int_{\Gamma}\left(\left(\widehat{\mathbf{v}}_{2}^{h}-\mathbf{v}\right) \cdot \mathbf{n}\right)^{2} d \Gamma\right) \\
\leq \frac{1}{h^{1+\epsilon_{0}}}\left\|u-\widehat{u}^{h}\right\|_{(1 / 2)\left(1+2 \delta-\epsilon_{0}\right)}^{2}+\frac{1}{h^{\epsilon_{1}}}\left\|\mathbf{v}-\widehat{\mathbf{v}}^{h}\right\|_{1-\frac{\epsilon_{1}}{2}}^{2} \\
\leq h^{2 s-2 \delta}\|u\|_{s+1}^{2}+\frac{1}{h^{\epsilon_{1}}} h^{2\left(s+\frac{\epsilon_{1}}{2}\right)}\|\mathbf{v}\|_{s+1}^{2} \leq C h^{2(s-\delta)}\left(\|u\|_{s+1}^{2}+\|\mathbf{v}\|_{s+1}^{2}\right)
\end{aligned}
$$

for $h$ sufficiently small. Combining (3.11)-(3.14) yields (3.10).

Remark. The conclusion of Theorem 3.2 is also valid for problems with homogeneous Newmann boundary conditions and mixed homogeneous boundary conditions.

Remark. Theorem 3.2 is a generalization of Theorem 5.1 of [8]. We merely require that $u \in \dot{H}^{s+1}(\Omega)$ and $\mathbf{v} \in \dot{\mathbf{V}}^{s+1}(\Omega)$, i.e., regularity within each subdomain and not across interfaces. Furthermore, we allow for the use of nonconforming elements in the sense that the finite element functions $u^{h} \in H^{h}$ need not belong to $H^{1}(\Omega)$.

4. Inhomogeneous Neumann boundary conditions. We now consider problem (3.1) with the homogeneous Dirchlet boundary condition replaced by an inhomogeneous Neumann boundary condition; i.e., we consider the problem

$$
\begin{gathered}
-\operatorname{div}\left(\mathbf{v}_{i}\right)+c_{i} u_{i}=f \quad \text { in } \Omega_{i}, \quad i=1,2, \\
A_{i} \nabla u_{i}-\mathbf{v}_{i}=0 \quad \text { in } \Omega_{i}, \quad i=1,2, \\
\left.\mathbf{v} \cdot \mathbf{n}\right|_{\Gamma_{0}}=g, \quad[u]_{\Gamma}=0, \quad \text { and } \quad[\mathbf{v} \cdot \mathbf{n}]_{\Gamma}=0 .
\end{gathered}
$$

Define the functional $\mathcal{K}(u, \mathbf{v} ; f, g)$ on $H \times V$ as follows. For $u \in H$ and $\mathbf{v} \in V$,

$$
\begin{aligned}
\mathcal{K}(u, \mathbf{v} ; f, g) & =\sum_{i=1}^{2}\left(\left\|-\operatorname{div}\left(\mathbf{v}_{i}\right)+c_{i} u_{i}-f\right\|_{0}^{2}+\left\|A_{i} \nabla u_{i}-\mathbf{v}_{i}\right\|_{0}^{2}\right) \\
& +\frac{1}{h^{1+\epsilon_{0}}} \int_{\Gamma}[u]_{\Gamma}^{2} d \Gamma+\frac{1}{h^{\epsilon_{1}}} \int_{\Gamma}[\mathbf{v} \cdot \mathbf{n}]_{\Gamma}^{2} d \Gamma+\frac{1}{h^{\epsilon_{2}}} \int_{\Gamma_{0}}(\mathbf{v} \cdot \mathbf{n}-g)^{2} d \Gamma .
\end{aligned}
$$


First we prove a coercivity property for $\mathcal{K}$; the result and its proof are similar to that of Proposition 3.1. Let $H^{h} \subset H$ and $\mathbf{V}^{h} \subset \mathbf{V}$ be finite-dimensional subspaces satisfying the approximation properties (3.4) and (3.5).

PROPOSITION 4.1. Let $\left(u^{h}, \mathbf{v}^{h}\right) \in H^{h} \times \mathbf{V}^{\mathbf{h}}$ with $u^{h}=\left(u_{1}^{h}, u_{2}^{h}\right)$ and $\mathbf{v}^{h}=\left(\mathbf{v}_{1}^{h}, \mathbf{v}_{2}^{h}\right)$ and $(u, \mathbf{v}) \in H(\Gamma, \Omega) \times \mathbf{V}(\Gamma, \Omega)$ with $u=\left(u_{1}, u_{2}\right)$ and $\mathbf{v}=\left(\mathbf{v}_{1}, \mathbf{v}_{2}\right)$. Then, for $h$ sufficiently small, there is a constant $C>0$ independent of $h$ such that

$$
\mathcal{K}\left(u-u^{h}, \mathbf{v}-\mathbf{v}^{h} ; 0,0\right) \geq C\left(\left\|u-u^{h}\right\|_{1}^{2}+\left\|\mathbf{v}-\mathbf{v}^{h}\right\|_{\text {div }}^{2}\right) .
$$

Proof. Define

$$
\begin{aligned}
\widehat{\mathcal{K}}(u, \mathbf{v})= & \sum_{i=1}^{2}\left(\left\|-c_{i}^{-1 / 2} \operatorname{div}\left(\mathbf{v}_{i}\right)+c_{i}^{1 / 2} u_{i}\right\|_{0}^{2}+\left\|A_{i}^{1 / 2} \nabla u_{i}-A_{i}^{-1 / 2} \mathbf{v}_{i}\right\|_{0}^{2}\right. \\
& +\frac{1}{h^{1+\epsilon_{0}}} \int_{\Gamma}[u]_{\Gamma}^{2} d \Gamma+\frac{1}{h^{\epsilon_{1}}} \int_{\Gamma}[\mathbf{v} \cdot \mathbf{n}]_{\Gamma}^{2} d \Gamma+\frac{1}{h^{\epsilon_{2}}} \int_{\Gamma_{0}}(\mathbf{v} \cdot \mathbf{n})^{2} d \Gamma .
\end{aligned}
$$

It is easy to see that $\mathcal{K}(\cdot ; 0,0)$ and $\widehat{\mathcal{K}}(\cdot)$ are equivalent, i.e., that there exist two positive constants $C_{1}$ and $C_{2}$ such that

$$
C_{1} \mathcal{K}(u, \mathbf{v} ; 0,0) \leq \widehat{\mathcal{K}}(u, \mathbf{v}) \leq C_{2} \mathcal{K}(u, \mathbf{v} ; 0,0)
$$

for all $u \in H$ and $\mathbf{v} \in \mathbf{V}$. Thus, it suffices to prove that

$$
\widehat{\mathcal{K}}\left(u-u^{h}, \mathbf{v}-\mathbf{v}^{h}\right) \geq C\left(\left\|u-u^{h}\right\|_{1}^{2}+\left\|\mathbf{v}-\mathbf{v}^{h}\right\|_{\text {div }}^{2}\right)
$$

for some constant $C>0$ and all $u \in H(\Gamma, \Omega)$ and $\mathbf{v} \in V(\Gamma, \Omega)$. Now, by the definition of $H(\Gamma, \Omega)$ and $V(\Gamma, \Omega)$,

$$
\begin{aligned}
& \widehat{\mathcal{K}}\left(u-u^{h}, \mathbf{v}-\mathbf{v}^{h}\right) \\
& =\sum_{i=1}^{2}\left(\left\|-c_{i}^{-1 / 2} \operatorname{div}\left(\mathbf{v}_{i}-\mathbf{v}_{i}^{h}\right)+c_{i}^{1 / 2}\left(u_{i}-u_{i}^{h}\right)\right\|_{0}^{2}+\left\|A_{i}^{1 / 2} \nabla\left(u_{i}-u_{i}^{h}\right)-A_{i}^{-1 / 2}\left(\mathbf{v}_{i}-\mathbf{v}_{i}^{h}\right)\right\|_{0}^{2}\right) \\
& +\frac{1}{h^{1+\epsilon_{0}}} \int_{\Gamma}\left|u_{1}^{h}-u_{2}^{h}\right|^{2} d \Gamma+\frac{1}{h^{\epsilon_{1}}} \int_{\Gamma}\left(\left(\mathbf{v}_{1}^{h}-\mathbf{v}_{2}^{h}\right) \cdot \mathbf{n}\right)^{2} d \Gamma+\frac{1}{h^{\epsilon_{2}}} \int_{\Gamma_{0}}\left(\mathbf{v} \cdot \mathbf{n}-\mathbf{v}^{h} \cdot \mathbf{n}\right)^{2} d \Gamma .
\end{aligned}
$$

Integrating by parts, one has

$$
\begin{aligned}
&\left\|-c_{i}^{-1 / 2} \operatorname{div}\left(\mathbf{v}_{i}-\mathbf{v}_{i}^{h}\right)+c_{i}^{1 / 2}\left(u_{i}-u_{i}^{h}\right)\right\|_{0}^{2}+\left\|A_{i}^{1 / 2} \nabla\left(u_{i}-u_{i}^{h}\right)-A_{i}^{-1 / 2}\left(\mathbf{v}_{i}-\mathbf{v}_{i}^{h}\right)\right\|_{0}^{2} \\
&=\left\|c_{i}^{-1 / 2} \operatorname{div}\left(\mathbf{v}_{i}-\mathbf{v}_{i}^{h}\right)\right\|_{0}^{2}+\left\|c_{i}^{1 / 2}\left(u_{i}-u_{i}^{h}\right)\right\|_{0}^{2}-2 \int_{\Omega_{i}} \operatorname{div}\left(\mathbf{v}_{i}-\mathbf{v}_{i}^{h}\right)\left(u_{i}-u_{i}^{h}\right) d \Omega \\
&+\left\|A_{i}^{1 / 2} \nabla\left(u_{i}-u_{i}^{h}\right)\right\|_{0}^{2}+\left\|A_{i}^{-1 / 2}\left(\mathbf{v}_{i}-\mathbf{v}_{i}^{h}\right)\right\|_{0}^{2}-2 \int_{\Omega_{i}} \nabla\left(u_{i}-u_{i}^{h}\right) \cdot\left(\mathbf{v}_{i}-\mathbf{v}_{i}^{h}\right) d \Omega \\
&=\left\|\operatorname{div}\left(\mathbf{v}_{i}-\mathbf{v}_{i}^{h}\right)\right\|_{0}^{2}+\left\|u_{i}-u_{i}^{h}\right\|_{0}^{2}+\left\|A_{i}^{1 / 2} \nabla\left(u_{i}-u_{i}^{h}\right)\right\|_{0}^{2} \\
&+\left\|A_{i}^{-1 / 2}\left(\mathbf{v}_{\mathbf{i}}-\mathbf{v}_{\mathbf{i}}^{\mathbf{h}}\right)\right\|_{0}^{2}-2 \int_{\Gamma}\left(u_{i}-u_{i}^{h}\right)\left(\mathbf{v}_{i}-\mathbf{v}_{i}^{h}\right) \cdot \mathbf{n} d \Gamma \\
&+2 \int_{\Gamma_{0}}\left(u-u^{h}\right)\left(\mathbf{v}-\mathbf{v}^{h}\right) \cdot \mathbf{n} d \Gamma .
\end{aligned}
$$


Hence,

$$
\begin{aligned}
\widehat{\mathcal{K}}(u- & \left.u^{h}, \mathbf{v}-\mathbf{v}^{h}\right) \\
\geq C & \sum_{i=1}^{2}\left(\left\|\operatorname{div}\left(\mathbf{v}_{i}-\mathbf{v}_{i}^{h}\right)\right\|_{0}^{2}+\left\|u_{i}-u_{i}^{h}\right\|_{0}^{2}+\left\|\nabla\left(u_{i}-u_{i}^{h}\right)\right\|_{0}^{2}+\left\|\mathbf{v}_{i}-\mathbf{v}_{i}^{h}\right\|_{0}^{2}\right) \\
& +2 \int_{\Gamma}\left(u_{1}-u_{1}^{h}\right)\left(\mathbf{v}_{1}-\mathbf{v}_{1}^{h}\right) \cdot \mathbf{n} d \Gamma-2 \int_{\Gamma}\left(u_{2}-u_{2}^{h}\right)\left(\mathbf{v}_{2}-\mathbf{v}_{2}^{h}\right) \cdot \mathbf{n} d \Gamma \\
& +2 \int_{\Gamma_{0}}\left(u-u^{h}\right)\left(\mathbf{v}-\mathbf{v}^{h}\right) \cdot \mathbf{n} d \Gamma+\frac{1}{h^{1+\epsilon_{0}}} \int_{\Gamma}\left(u_{1}^{h}-u_{2}^{h}\right)^{2} d \Gamma \\
& +\frac{1}{h^{\epsilon_{1}}} \int_{\Gamma}\left(\left(\mathbf{v}_{1}^{h}-\mathbf{v}_{2}^{h}\right) \cdot \mathbf{n}\right)^{2} d \Gamma+\frac{1}{h^{\epsilon_{2}}} \int_{\Gamma_{0}}\left(\mathbf{v} \cdot \mathbf{n}-\mathbf{v}^{h} \cdot \mathbf{n}\right)^{2} d \Gamma \\
=I+ & I I+I I I+I V,
\end{aligned}
$$

where

$$
\begin{aligned}
I & =C \sum_{i=1}^{2}\left(\left\|\operatorname{div}\left(\mathbf{v}_{i}-\mathbf{v}_{i}^{h}\right)\right\|_{0}^{2}+\left\|u_{i}-u_{i}^{h}\right\|_{0}^{2}+\left\|\nabla\left(u-u_{h}\right)\right\|_{0}^{2}+\left\|\mathbf{v}_{i}-\mathbf{v}_{i}^{h}\right\|_{0}^{2}\right), \\
I I & =2\left(\int_{\Gamma}\left(u_{1}-u_{1}^{h}\right)\left(\mathbf{v}_{1}-\mathbf{v}_{1}^{h}\right) \cdot \mathbf{n} d \Gamma-\int_{\Gamma}\left(u_{2}-u_{2}^{h}\right)\left(\mathbf{v}_{2}-\mathbf{v}_{2}^{h}\right) \cdot \mathbf{n} d \Gamma\right), \\
I I I= & 2 \int_{\Gamma_{0}}\left(u-u^{h}\right)\left(\mathbf{v}-\mathbf{v}^{h}\right) \cdot \mathbf{n} d \Gamma, \\
I V= & \frac{1}{h^{1+\epsilon_{0}}} \int_{\Gamma}\left(u_{1}^{h}-u_{2}^{h}\right)^{2} d \Gamma \\
& \quad+\frac{1}{h^{\epsilon_{1}}} \int_{\Gamma}\left(\left(\mathbf{v}_{1}^{h}-\mathbf{v}_{2}^{h}\right) \cdot \mathbf{n}\right)^{2} d \Gamma+\frac{1}{h^{\epsilon_{2}}} \int_{\Gamma_{0}}\left(\mathbf{v} \cdot \mathbf{n}-\mathbf{v}^{h} \cdot \mathbf{n}\right)^{2} d \Gamma .
\end{aligned}
$$

By the proof of Theorem 3.1 we have that for $\epsilon>0$,

$$
|I I| \leq C \epsilon\left\|\mathbf{v}-\mathbf{v}^{h}\right\|_{\text {div }}^{2}+\frac{1}{h \epsilon}\left\|u_{1}^{h}-u_{2}^{h}\right\|_{0, \Gamma}^{2}+\frac{1}{\epsilon}\left\|\mathbf{v}_{1}^{h}-\mathbf{v}_{2}^{h}\right\|_{0, \Gamma}^{2}+\epsilon\left\|u-u_{h}\right\|_{1}^{2} .
$$

Using the Schwartz inequality we have that

$$
|I I I|=2\left|\int_{\Gamma_{0}}\left(u-u^{h}\right)\left(\mathbf{v} \cdot \mathbf{n}-\mathbf{v}^{h} \cdot \mathbf{n}\right) d \Gamma\right| \leq \epsilon_{3} \int_{\Gamma_{0}}\left(u-u^{h}\right)^{2} d \Gamma+\frac{1}{\epsilon_{3}} \int_{\Gamma_{0}}\left(\mathbf{v} \cdot \mathbf{n}-\mathbf{v}^{h} \cdot \mathbf{n}\right)^{2} d \Gamma
$$

for $\epsilon_{3}>0$. Hence

$$
\begin{aligned}
\widehat{\mathcal{K}}\left(u-u^{h}, \mathbf{v}-\mathbf{v}^{h}\right) & \geq\left(C_{1}-\epsilon-\epsilon_{3}\right)\left\|u-u^{h}\right\|_{1}^{2}+\left(C_{2}-\epsilon\right)\left\|\mathbf{v}-\mathbf{v}^{h}\right\|_{\text {div }}^{2} \\
& +\left(\frac{1}{h^{1+\epsilon_{0}}}-\frac{1}{h \epsilon}\right) \int_{\Gamma}\left|u_{1}^{h}-u_{2}^{h}\right|^{2} d \Gamma+\left(\frac{1}{h^{\epsilon_{1}}}-\frac{1}{\epsilon}\right) \int_{\Gamma}\left(\left(\mathbf{v}_{1}^{h}-\mathbf{v}_{2}^{h}\right) \cdot \mathbf{n}\right)^{2} d \Gamma \\
& +\left(\frac{1}{h^{\epsilon_{2}}}-\frac{1}{\epsilon_{3}}\right) \int_{\Gamma_{0}}\left(\mathbf{v} \cdot \mathbf{n}-\mathbf{v}^{h} \cdot \mathbf{n}\right)^{2} d \Gamma .
\end{aligned}
$$

We first choose $\epsilon$ and $\epsilon_{3}$ small enough so that $C_{i}-\epsilon-\epsilon_{3}>0$. Then, for this fixed $\epsilon$, we let $h_{0}$ be sufficiently small so that $h_{0}^{1+\epsilon_{0}} \leq h \epsilon, h_{0}^{\epsilon_{1}}<\epsilon$, and $h_{0}^{\epsilon_{2}}<\epsilon_{3}$. Thus, for $0>h>h_{0}$, we obtain

$$
\widehat{\mathcal{K}}\left(u-u^{h}, \mathbf{v}-\mathbf{v}^{h}\right) \geq C\left(\left\|u-u^{h}\right\|_{1}^{2}+\left\|\mathbf{v}-\mathbf{v}^{h}\right\|_{\text {div }}^{2}\right) .
$$

The proof is complete. 
Assume that $\left(u_{*}^{h}, \mathbf{v}_{*}^{h}\right)$ is the solution of the following problem:

$$
\mathcal{K}\left(u_{*}^{h}, \mathbf{v}_{*}^{h} ; f, g\right)=\min _{u^{h} \in H^{h}, \mathbf{v}^{h} \in \mathbf{V}^{h}} \mathcal{K}\left(u^{h}, \mathbf{v}^{h} ; f, g\right) .
$$

We have the following error estimate.

THEOREM 4.2. Let $s>0$. Assume that the solutions $u$ and $\mathbf{v}$ of (4.1)-(4.3) satisfy $u \in \dot{H}^{s+1}(\Omega)$ and $\mathbf{v} \in \dot{V}^{s+1}(\Omega)$. Then, for $h$ sufficiently small and for $0<\epsilon_{1}, \epsilon_{2} \leq 1$, and any $\delta>\epsilon_{0}>0$, there exists a constant $C>0$ such that

$$
\left\|u-u_{*}^{h}\right\|_{1}+\left\|\mathbf{v}-\mathbf{v}_{*}^{h}\right\|_{\text {div }} \leq C h^{s-\delta}\left(\|u\|_{s+1}+\|\| \mathbf{v} \|_{s+1}\right) .
$$

Proof. By the approximation properties (3.4) and (3.5), there exist $\widehat{u}^{h} \in H^{h}$ and $\widehat{\mathbf{v}}^{h} \in \mathbf{V}^{h}$ such that

$$
\left\|u-\widehat{u}^{h}\right\|_{1} \leq C h^{s}\|u\|_{s+1}
$$

and

$$
\left\|\mathbf{v}-\widehat{\mathbf{v}}^{h}\right\|_{1} \leq C h^{s}\|\mathbf{v}\|_{s+1} .
$$

By Proposition 4.1 and the definition of $u_{*}^{h}$ and $\mathbf{v}_{*}^{h}$ we have that

$$
\begin{aligned}
& \|\| u-u_{*}^{h}\left\|_{1}^{2}+\right\| \mathbf{v}-\mathbf{v}_{*}^{h} \|_{\text {div }}^{2} \\
& \leq C \mathcal{K}\left(u-u_{*}^{h}, \mathbf{v}-\mathbf{v}_{*}^{h} ; 0,0\right)=C \mathcal{K}\left(u_{*}^{h}, \mathbf{v}_{*}^{h} ; f, g\right) \\
& \leq C \mathcal{K}\left(\widehat{u}^{h}, \widehat{\mathbf{v}}^{h} ; f, g\right)=C \mathcal{K}\left(u-\widehat{u}^{h}, \mathbf{v}-\widehat{\mathbf{v}}^{h} ; 0,0\right) \\
& \leq\|\| u-\widehat{u}^{h}\left\|_{1}^{2}+\right\| \mathbf{v}-\widehat{\mathbf{v}}^{h} \|_{\text {div }}^{2}+\frac{1}{h^{1+\epsilon_{0}}} \int_{\Gamma}\left(\widehat{u}_{1}^{h}-\widehat{u}_{2}^{h}\right)^{2} d \Gamma \\
& \quad+\frac{1}{h^{\epsilon_{1}}} \int_{\Gamma}\left(\left(\widehat{\mathbf{v}}_{1}^{h}-\widehat{\mathbf{v}}_{2}^{h}\right) \cdot \mathbf{n}\right)^{2} d \Gamma+\frac{1}{h^{\epsilon_{2}}} \int_{\Gamma_{0}}\left(\left(\widehat{\mathbf{v}}^{h}-\mathbf{v}\right) \cdot \mathbf{n}\right)^{2} d \Gamma .
\end{aligned}
$$

From the proof of Theorem 3.2, we have that

$$
\begin{aligned}
& \frac{1}{h^{1+\epsilon_{0}}} \int_{\Gamma}\left(\widehat{u}_{1}^{h}-\widehat{u}_{2}^{h}\right)^{2} d \Gamma+\frac{1}{h^{\epsilon_{1}}} \int_{\Gamma}\left(\left(\widehat{\mathbf{v}}_{1}^{h}-\widehat{\mathbf{v}}_{2}^{h}\right) \cdot \mathbf{n}\right)^{2} d \Gamma \\
& \quad \leq h^{2 s-2 \delta}\|\| u\left\|_{s+1}^{2}+\frac{1}{h^{\epsilon_{1}}} h^{2\left(s+\frac{\epsilon_{1}}{2}\right)}\right\|\|\mathbf{v}\|_{s+1}^{2} \leq C h^{2(s-\delta)}\left(\|u\|_{s+1}^{2}+\|\mathbf{v}\|_{s+1}^{2}\right) .
\end{aligned}
$$

Using trace theorems we have that

$$
\begin{aligned}
\frac{1}{h^{\epsilon_{2}}} \int_{\Gamma_{0}}\left(\mathbf{v} \cdot \mathbf{n}-\widehat{\mathbf{v}}^{h} \cdot \mathbf{n}\right)^{2} d \Gamma & \leq \frac{1}{h^{\epsilon_{2}}}\left\|\mathbf{v}-\widehat{\mathbf{v}}^{h}\right\|_{1-\frac{\epsilon_{2}}{2}}^{2} \\
& \leq \frac{1}{h^{\epsilon_{2}}} h^{2\left(s+\frac{\epsilon_{2}}{2}\right)}\|\mathbf{v}\|_{s+1}^{2}=C h^{2 s}\|\mathbf{v}\|_{s+1}^{2}
\end{aligned}
$$

for $h$ sufficiently small. Substituting (4.9) and (4.10) into (4.8), we obtain (4.7). 


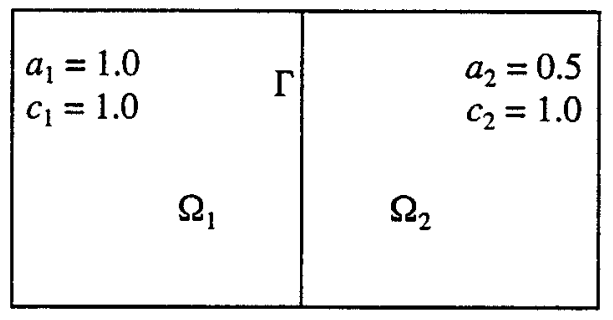

FIG. 1. Interface problem used in computational example.

5. Numerical results. In this section we report the results of computations which illustrate our method and error analysis. We take for the domain the rectangle $\Omega=(0,2) \times(0,1)$. The interface occurs at $x=1$ so that $\Omega_{1}=(0,1) \times(0,1)$ and $\Omega_{2}=(1,2) \times(0,1)$. In $(2.1)-(2.3), A_{i}=\operatorname{diag}\left(a_{i}, a_{i}\right)$ with $a_{1}=1$ and $a_{2}=1 / 2$ and $c_{1}=c_{2}=1 ;$ see Figure 1 .

For the exact solution, we choose

$$
u_{1}(x, y)=\sin (\pi x) \sin (\pi y), \quad(x, y) \in \Omega_{1}=(0,1) \times(0,1)
$$

and

$$
u_{2}(x, y)=-\sin (2 \pi x) \sin (\pi y), \quad(x, y) \in \Omega_{2}=(1,2) \times(0,1) .
$$

The right-hand sides $f_{1}$ and $f_{2}$ in (2.1) are then determined from this choice for $\left(A_{1}, c_{1}, u_{1}\right)$ and $\left(A_{2}, c_{2}, u_{2}\right)$, respectively. Note that the global solution merely belongs to $H^{1}(\Omega)$. We choose $\epsilon_{0}=2 / 3$ and $\epsilon_{1}=3 / 4$ in the functional (3.4).

Standard techniques of the calculus of variations may be used to deduce that any solution $\left(u_{*}^{h}, \mathbf{v}_{*}^{h}\right)$ of $(3.8)$ necessarily satisfies the variational problem: find $\left(u_{*}^{h}, \mathbf{v}_{*}^{h}\right) \in$ $H^{h} \times V^{h}$ such that

$$
B\left(\left(u_{*}^{h}, \mathbf{v}_{*}^{h}\right),\left(\widetilde{u}^{h}, \widetilde{\mathbf{v}}^{h}\right)\right)=F\left(\left(\widetilde{u}^{h}, \widetilde{\mathbf{v}}^{h}\right)\right) \quad \forall\left(\widetilde{u}^{h}, \widetilde{\mathbf{v}}^{h}\right) \in H^{h} \times V^{h},
$$

where, for $u^{h}=\left(u_{1}^{h}, u_{2}^{h}\right), \mathbf{v}^{h}=\left(\mathbf{v}_{1}^{h}, \mathbf{v}_{2}^{h}\right), \widetilde{u}^{h}=\left(\widetilde{u}_{1}^{h}, \widetilde{u}_{2}^{h}\right), \mathbf{v}^{h}=\left(\widetilde{\mathbf{v}}_{1}^{h}, \widetilde{\mathbf{v}}_{2}^{h}\right)$, we have

$$
\begin{aligned}
B\left(\left(u^{h}, \mathbf{v}^{h}\right),\left(\widetilde{u}^{h}, \widetilde{\mathbf{v}}^{h}\right)\right) & \\
= & \sum_{i=1}^{2}\left(\left(\operatorname{div}\left(\mathbf{v}_{i}^{h}\right)+c_{i} u_{i}, \operatorname{div}\left(\widetilde{\mathbf{v}}_{i}^{h}\right)+c_{i} \widetilde{u}_{i}^{h}\right)_{\Omega_{i}}+\left(A_{i} \nabla u_{i}^{h}-\mathbf{v}_{i}^{h}, A_{i} \nabla \widetilde{u}_{i}^{h}-\widetilde{\mathbf{v}}_{i}^{h}\right)_{\Omega_{i}}\right) \\
& +\frac{1}{h^{1+\epsilon_{0}}}\left(u_{1}^{h}-u_{2}^{h}, \widetilde{u}_{1}^{h}-\widetilde{u}_{2}^{h}\right)_{\Gamma}+\frac{1}{h^{\epsilon_{1}}}\left(\left(\mathbf{v}_{1}^{h}-\mathbf{v}_{2}^{h}\right) \cdot \mathbf{n},\left(\widetilde{\mathbf{v}}_{1}^{h}-\widetilde{\mathbf{v}}_{2}^{h}\right) \cdot \mathbf{n}\right)_{\Gamma}
\end{aligned}
$$

and

$$
F\left(\left(\widetilde{u}^{h}, \widetilde{\mathbf{v}}^{h}\right)\right)=\sum_{i=1}^{2}\left(f_{i},-\operatorname{div}\left(\widetilde{\mathbf{v}}_{i}^{h}\right)+\widetilde{u}_{i}^{h}\right)_{\Omega_{i}} .
$$

Here, $(\cdot, \cdot)_{\Omega_{i}}$ and $(\cdot, \cdot)_{\Gamma}$ denote the $L^{2}\left(\Omega_{i}\right)$ and $L^{2}(\Gamma)$ inner products, respectively. 

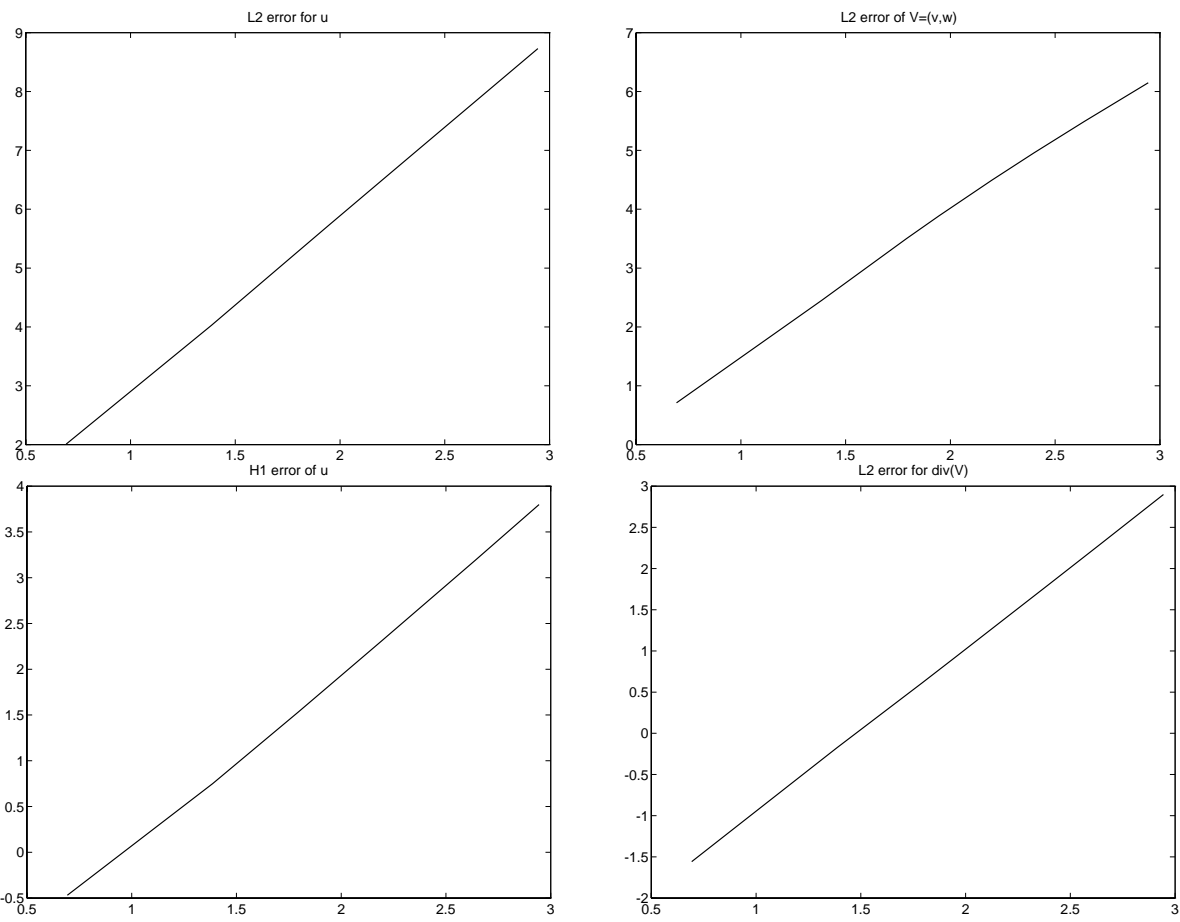

FIG. 2. Negative of logarithm of error vs. $-\log (h)$.

TABLE 1

Rates of convergence.

\begin{tabular}{|c|c|c|}
\hline Function & $L^{2}$ error & $H^{1}$ error \\
\hline$u$ & 3.315 & 1.837 \\
\hline$v$ & 2.321 & 1.214 \\
\hline$w$ & 2.138 & 1.038 \\
\hline $\operatorname{div} \mathbf{~ V}$ & 1.898 & - \\
\hline
\end{tabular}

For our numerical results, globally continuous piecewise quadratic finite element functions based on uniform triangulations of $\Omega_{i}, i=1,2$, were used for all unknowns, i.e., $u_{i}^{h}$ and the components of $\mathbf{v}_{i}^{h}, i=1,2$. The nodes of the triangulations of $\Omega_{1}$ and $\Omega_{2}$ coincide on the interface $\Gamma$. Hence, we expect that convergence rates will be determined according to (3.9) with $s=3$.

Figure 2 displays the $L_{2}$ error of the approximate solutions for $u=\left(u_{1}, u_{2}\right)$, $\mathbf{v}=\left(A_{1} \nabla u_{1}, A_{2} \nabla u_{2}\right)$, the error of $u$ in the $H^{1}$ seminorm, and the $L_{2}$ norm error of div v. In Table 1, we list the rates of convergence estimated by linear regression. These convergence rates match our error estimates in section 3.

Remark. From Table 1, we see that the $L^{2}$ error in the approximation to $u$ is one order higher than that for its derivative.

Remark. As shown in [9] and [13], if $\|\operatorname{curl} \mathbf{v}\|^{2}$ is added to the standard leastsquares functional, then the optimal error estimate in the $H^{1}$-norm for $\mathbf{v}$ may be achieved. Also, the error for $\mathbf{v}$ in the $L^{2}$ norm of is one order higher. Our numerical experience (see Figure 3) indicates that if we add $\|\operatorname{curl} \mathbf{v}\|^{2}$ to the functional (3.4), then the $H^{1}$ error in the approximation $\mathbf{v}$ is seemingly better, but the $L^{2}$ error is not improved. 

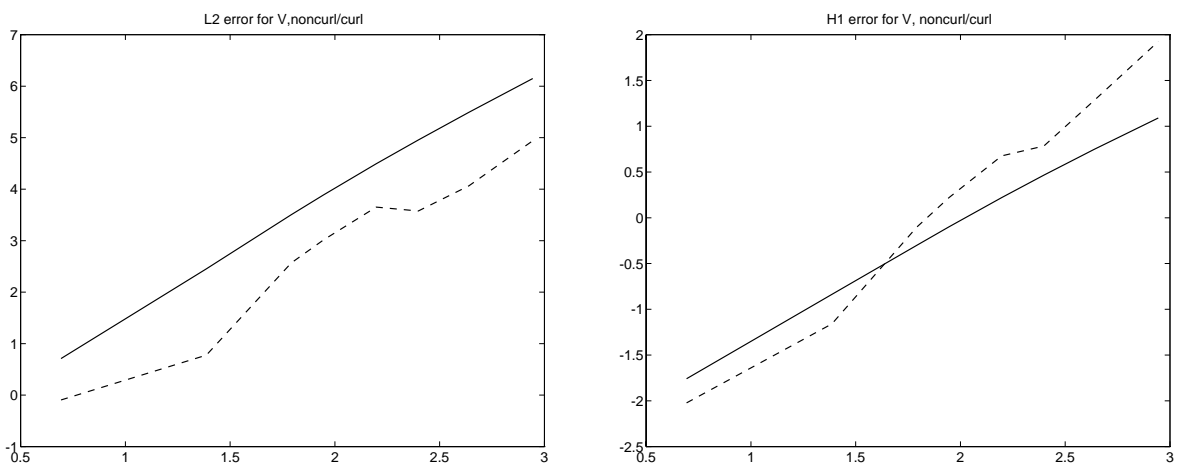

FIG. 3. Negative of logrithm of error vs. $-\log (h)$. Solid line: without curl term in functional; dashed line: with curl term in functional.

Acknowledgment. The authors thank Steve McCormick for the many suggestions that materially improved this paper.

\section{REFERENCES}

[1] A. Aziz, R. Kellog, And A. Stephens, Least-squares methods for elliptic systems, Math. Comp., 44 (1985), pp. 53-70.

[2] I. BABUSKA, The finite element method for elliptic equations, Computing, (1970), pp. 207-213.

[3] I. BABUSKA AND A. AzIz, Survey lectures on the mathematical foundations of the finite element method, in The Mathematical Foundations of the Finite Element Method with Applications to Partial Differential Equations, A. Aziz, ed., Academic, New York, 1973, pp. 3-359.

[4] P. Bochev, Z. CAi, T. Manteuffel, And S. MCCormick, First-order system least-squares for the Navier-Stokes equations, in Proc. 7th Copper Mountain Conf. on Multigrid Methods, to appear.

[5] P. Bochev And M. Gunzburger, Accuracy of least-squares methods for the Navier-Stokes equations, Comput. Fluids, 22 (1993), pp. 549-563.

[6] P. Bochev And M. Gunzburger, Analysis of least-squares finite element methods for the Stokes equations, Math. Comp., 63 (1994), pp. 479-506.

[7] P. Bochev And M. GunzBurger, Least squares methods for the velocity-pressure-stress formulation of the stokes equations, Comp. Meth. Appl. Mech. Engrg., 126 (1995), pp. 267287.

[8] Z. Cai, R. Lazarov, T. Manteuffel, And S. MCCormick, First-order system least-squares for partial differential equations: Part I, SIAM J. Numer. Anal., 31 (1994), pp. 1785-1799.

[9] Z. Cai, T. Manteuffel, ANd S. MCCoRmick, First-order system least-squares for partial differential equations: Part II, SIAM J. Numer. Anal., 34 (1997), pp. 425-454.

[10] Z. Cai, T. Manteuffel, And S. MCCormick, First-order system least-squares for the Stokes equations with application to linear elasticity, SIAM J. Numer. Anal., 34 (1997), pp. 17271741.

[11] C.-L. Chang, An error estimate of the least squares finite element method for the Stokes problem in three dimensions, Math. Comp., 63 (1994), pp. 41-50.

[12] J. Deang And M. Gunzburger, Issues related to least-squares finite element methods for the Stokes equations, SIAM J. Sci. Comp., to appear.

[13] B.-N. JiAng And L. Povinelli, Optimal least-squares finite element method for elliptic problems, Comput. Methods Appl. Mech. Engrg., 102 (1993), pp. 199-212.

[14] D. Lefevre, J. Peraire, And K. Morgan, Least-squares finite element solution of compressible and incompressible flows, Internat. J. Numer. Methods Heat Fluid Flow, 2 (1992), pp. 99-113.

[15] T. Manteuffel, S. McCormick, and G. Starke, First-order system of least-squares for second order elliptic problems with discontinuous coefficients, to appear.

[16] Z. Seftel, A general theory of boundary value problems for elliptic systems with discontinuous doefficients, Ukrainian Math. Z., 18 (1966), pp. 132-136. 